\title{
Prognostic and predictive factors associated with ipilimumab- related adverse events: a retrospective analysis of 11 NCI- sponsored phase I clinical trials
}

\author{
Aman Chauhan ${ }^{1}$, Tanvir Kabir ${ }^{2}$, Jianrong Wu${ }^{3}$, Jing Wei ${ }^{4}$, Mary Cook ${ }^{1}$ and Charles \\ A. Kunos ${ }^{5}$ \\ ${ }^{1}$ Division of Medical Oncology, University of Kentucky, Lexington, KY, USA \\ ${ }^{2}$ College of Medicine, University of Kentucky, Lexington, KY, USA \\ ${ }^{3}$ Markey Cancer Center, Biostatistics and Bioinformatics Shared Resource Facility, University of Kentucky, Lexington, KY, USA \\ ${ }^{4}$ Department of Statistics, University of Kentucky, Lexington, KY, USA \\ ${ }^{5}$ Cancer Therapy Evaluation Program, National Cancer Institute, Bethesda, MD, USA \\ Correspondence to: Aman Chauhan, email: Aman.Chauhan@uky.edu
}

Keywords: immunotherapy; toxicity; co-administered agents; metastatic melanoma; immune checkpoint inhibitors Received: December 16, $2019 \quad$ Accepted: March 14, $2020 \quad$ Published: April 21, 2020

Copyright: Chauhan et al. This is an open-access article distributed under the terms of the Creative Commons Attribution License 3.0 (CC BY 3.0), which permits unrestricted use, distribution, and reproduction in any medium, provided the original author and source are credited.

\section{ABSTRACT}

Background: We review factors impacting ipilimumab-associated adverse events through the experience from National Cancer Institute (NCI)-sponsored phase I immunotherapy clinical trials.

Materials and Methods: Attributable ipilimumab-related adverse events from NCI-sponsored phase I immunotherapy clinical trials were queried retrospectively by anonymized patient experience reports for observed adverse events like decreased hematological cell counts, blood electrolytes or proteins, or reduced patient performance status. The prevalence of ipilimumab-related toxicity was associated by patient to the duration of ipilimumab exposure, radiographic responses, progressionfree survival, and overall survival.

Results: 373 patients from 11 phase 1 ipilimumab clinical trials were analyzed. Patients experiencing at least one grade 3 or 4 adverse event associated with observed radiographic response were included. The average number of grade $3 / 4$ adverse events in responders was 1.167 versus 0.645 in non-responders $(p=$ 0.001 ). Patient performance status did not significantly impact observed toxicity grade. Pretherapy lymphocyte count or chemistries were not associated with ipilimumab-associated toxicity. The number of agents combined with ipilimumab on trial was associated with average number of grade 3/4 toxicities-ipilimumab monotherapy $(0.631)$ versus ipilimumab +1 agent $(0.877)$ versus ipilimumab + 2 agents $(1.408)(p=0.014)$. Number of low grade (grade $1 / 2)$ toxicities was associated with duration of treatment, Pearson correlation coefficient $r=0.456$ $(p<0.0001)$; whereas the number of high grade (grade $3 / 4)$ toxicities was not, $r=0.032(p=0.546)$.

Conclusions: Ipilimumab-attributed grade $3 / 4$ toxicity was associated with therapeutic response. The number of co-administered agents added to ipilimumab significantly raised the likelihood of toxicity. Extended duration of treatment increased the incidence of low but not high-grade toxicity. 


\section{INTRODUCTION}

Cancer clinical trials offer the best data to study, treat, and cure cancer. Over 1,400 clinical trials are actively recruiting cancer patients to treatments that involve immunotherapy with at least one anticancer agent, with ipilimumab studied in about 500 of those trials [1]. The immune checkpoint inhibitor ipilimumab inhibits cytotoxic T-lymphocyte associated protein 4 (CTLA4 or CD152) and renders exposed cancer cells sensitive to T-cell mediated destruction [2]. The number of ipilimumab users in cancer clinics has expanded substantially and ipilimumab currently serves as one of the most often combined cancer immunotherapy agents against cancer. The best known ipilimumab-associated adverse event or toxicity experience arises from human clinical trials evaluating it against skin cancer melanoma [3]. Limited data are available for biological or clinical markers that might predict ipilimumab-attributed toxicity.

In this retrospective article, we collect and analyze ipilimumab-attributed toxicity from 11 National Cancer Institute (NCI)-sponsored phase 1 trials evaluating the agent in a variety of adult solid and hematological cancers with the aims of associating patient and clinical factors with engendered ipilimumab-attributed toxicity and treatment outcomes.

\section{RESULTS}

Data from 373 patients from 11 phase I ipilimumab clinical trials were available for the analysis. Median age of the study cohort was 55 years and $68 \%(n=253)$ were males. ECOG performance status was available in all but nine patients. 222 patients $(61 \%)$ had ECOG performance status of 0 , and $142(38 \%)$ had ECOG performance status of 1 or 2 at the time of enrollment. No significant differences in grade 1 or 2 (here deemed low grade), or, grade 3 or 4 (high grade) adverse events were identified in the study cohorts based on ECOG performance status (0 versus 1 or 2). Patients with ECOG performance status 3 or 4 were not eligible for these clinical trials.

We also looked into association between toxicity and pretreatment albumin (a surrogate for baseline nutritional status), lactate dehydrogenase (LDH; a surrogate marker for tumor bulk) and lymphocyte count (hypothesis being higher baseline lymphocyte count might result in higher autoimmune toxicities). Pretreatment albumin was available for analysis in 92 patients, pretreatment LDH was available in 90 patients and pretreatment lymphocyte count was available in 88 patients. Pretreatment lymphocyte count, LDH or albumin was not predictive of increased ipilimumab associated toxicity (Table 1).

Radiological responses were correlated with the grade of observed adverse events. Best radiological responses were analyzed and divided between two cohorts-stable disease $(n=273)$ versus complete or partial response $(\mathrm{CR} / \mathrm{PR})(n=54)$. Grade 3 and 4 adverse events were associated with better radiological response $(p=0.001)$. The average number of grade 3 or 4 adverse events in the CR/PR cohort was 1.167 , versus 0.645 in the non-responder cohort.

Of all patients, $243(65 \%)$ were treated with only ipilimumab, 81 patients $(22 \%)$ were treated with two agents including ipilimumab and 49 patients (13\%) were treated with three agents including ipilimumab. Ipilimumab-associated grade 3 and 4 toxicity was directly associated with the co-administered number of study agents. The average number of grade 3 and 4 adverse events was 0.631 after ipilimumab alone, 0.877 after ipilimumab plus one other agent and 1.408 after ipilimumab plus two agents $(p=0.014)$.

Lastly, we looked into the association of lowand high-grade toxicities with regard to duration of ipilimumab treatment. Data on duration of therapy were available on 366 patients. Low grade (grade 1 or 2) toxicity was associated with the duration of treatment. The longer patients stayed on treatment, the higher the incidence of grade 1 or 2 adverse events as suggested by Pearson correlation coefficient $(r=0.456 ; p<0.0001)$. The number of high-grade (grade 3 or 4 ) toxicity was not associated with the duration of treatment with ipilimumab $(r=0.032 ; p=0.546)$. Grade 3 or 4 adverse events were noted in patients with both minimal exposure to drug as well as those who were on ipilimumab for a long duration (See Table 1).

\section{DISCUSSION}

Although numerous studies evaluate the efficacy and toxicity of ipilimumab in melanoma, less data are available on ipilimumab-associated adverse events in other types of solid and hematological tumors. Furthermore, data regarding clinical and serum markers that may predict such adverse events has been limited. From among 373 patients participating in 11 ipilimumab immunotherapy trials sponsored by the NCI to treat various cancers, our study found most importantly and not unexpectedly that ipilimumab-attributed toxicity associated with the number of co-administered agents, and the prevalence of toxicity increased according to the number of agents in the ipilimumab combination (See Table 2). This finding can be put into context with a randomized double-blind phase III trial treating 945 metastatic melanoma patients taking/ using/administering either ipilimumab and nivolumab together, or either ipilimumab or nivolumab individually/ alone.

The prevalence of grade 3 or 4 adverse events was higher for the combination as compared to either agent alone. In this trial, $55 \%$ of patients treated with both ipilimumab and nivolumab experienced grade 3 or 4 adverse events, whereas $27 \%$ of patients after ipilimumab alone and $16 \%$ of patients after nivolumab had grade 3 or 
Table 1: Patient demographic and prognostic variables, toxicity and outcome results

\begin{tabular}{|c|c|c|c|}
\hline Variable & $N(\%)$ & All grade Mean (STD) & Grade 3 + Grade 4 Mean (STD) \\
\hline Age (continuous) & $N=373$ & $r=0.089(p=0.084)$ & $r=0.062(p=0.232)$ \\
\hline Mean & 52 & & \\
\hline Median & 55 & & \\
\hline \multicolumn{4}{|l|}{ Age (categorical) } \\
\hline$\leq 55$ & 187 & $6.02(6.15)$ & $0.717(1.25)$ \\
\hline$>55$ & 186 & $6.69(5.15)(p=0.029)$ & $0.833(1.38)(p=0.398)$ \\
\hline \multicolumn{4}{|l|}{ Sex } \\
\hline Female & $120(32 \%)$ & $6.01(6.83)$ & $0.625(1.19)$ \\
\hline Male & $253(68 \%)$ & $6.52(5.31)(p=0.103)$ & $0.846(1.37)(p=0.111)$ \\
\hline \multicolumn{4}{|l|}{ ECOG PS } \\
\hline 0 & $222(61 \%)$ & $6.76(5.72)$ & $0.748(1.31)$ \\
\hline 1 and 2 & $142(38 \%)$ & $5.78(5.67)(p=0.048)$ & $0.803(1.370)(p=0.590)$ \\
\hline Missing (excluded) & $9(2 \%)$ & & \\
\hline \multicolumn{4}{|l|}{ Number of agents } \\
\hline 1 & $243(65 \%)$ & $5.61(5.57)$ & $0.613(1.05)$ \\
\hline 2 & $81(22 \%)$ & $6.62(5.86)$ & $0.877(1.54)$ \\
\hline 3 & $49(13 \%)$ & $9.59(4.73)$ & $1.408(1.84)$ \\
\hline Overall chi-square test & & $p<0.0001$ & $p=0.0136$ \\
\hline Pairwise test (1 vs 2) & & $p=0.146$ & $p=0.3174$ \\
\hline Pairwise test (1 vs 3 ) & & $p<0.0001$ & $p=0.0035$ \\
\hline Pairwise test (2 vs 3 ) & & $p=0.0002$ & $p=0.085$ \\
\hline Pre-treatment albumin & 92 & $r=0.157(p=0.136)$ & $r=0.142(p=0.178)$ \\
\hline Pre-treatment LDH & 90 & $r=-0.289(p=0.006)$ & $r=-0.132(p=0.213)$ \\
\hline Pre-treatment lymphocyte count & 88 & $r=-0.077(p=0.475)$ & $r=-0.028(p=0.797)$ \\
\hline \multicolumn{4}{|l|}{ Best response } \\
\hline No response & 273 & 6.01 & 0.645 \\
\hline $\mathrm{CR}+\mathrm{PR}$ & 54 & $9.27(p<0.0001)$ & $1.167(p=0.001)$ \\
\hline \multicolumn{4}{|l|}{ Disease progression } \\
\hline Yes & 192 & 5.01 & 0.578 \\
\hline No & 135 & $8.74(p<0.0001)$ & $0.948(p=0.039)$ \\
\hline Duration of therapy & 366 & $r=0.456(p<0.0001)$ & $r=-0.032(p=0.546)$ \\
\hline
\end{tabular}

4 adverse events. Despite an increase in overall survival after ipilimumab-nivolumab treatment, the relatively high prevalence of severe toxicities after combination treatment became a barrier to treatment. It is noteworthy that the resolution of severe immunotherapy-related adverse events was similar in all three patient treatment groups.

Various studies involving checkpoint inhibitor combination therapy are consistent with these findings. A retrospective analysis of approximately 7700 metastatic melanoma patients treated with ipilimumab and/or nivolumab showed that when used concurrently, their toxicity profile combines adverse events from each individual drug [4]. Another retrospective analysis of a phase I, II and III trial consisting of 448 patients with advanced melanoma treated with ipilimumab and nivolumab demonstrated higher levels of grade 3 and 4 adverse events (55\%) compared to either agent alone [5]. Greater rates of toxicity have been reported even when immune checkpoint inhibitors have been used sequentially. For example, in a retrospective analysis of phase III studies, 40 patients with metastatic melanoma received nivolumab or pembrolizumab and then upon progression received ipilimumab [6]. $35 \%$ of patients from this phase III study developed grade 3-5 immune related adverse events. Three patients (7\%) developed grade 3-5 pneumonitis leading to the death of one patient. A randomized phase II study consisting of 140 patients found clinical benefit when nivolumab was used sequentially with ipilimumab; however, it was also noted that the sequential combination was associated with a greater frequency of adverse events [7].

When testing ipilimumab concurrently with cytotoxic chemotherapy, the results were similar. In a phase III trial evaluating ipilimumab with or without dacarbazine in 502 patients with untreated metastatic melanoma, grade 3 and 4 adverse events were more 
frequent in ipilimumab plus dacarbazine (56\%) compared to dacarbazine plus placebo (28\%) [8]. The pattern of immune-related adverse events differed, with approximately $20 \%$ more hepatotoxicity and $2 \%$ less colitis in the ipilimumab plus dacarbazine group. In a phase III study treating 954 small cell lung cancer patients with either ipilimumab with etoposide and platinum or with chemotherapy alone, rates and severity of treatmentrelated adverse effects were similar between groups except more frequent diarrhea, rash and colitis occurred in the group treated with ipilimumab plus chemotherapy [9]. There was significantly higher treatment related discontinuation in the group with ipilimumab $(18 \%)$ compared to the control group (2\%). Even when evaluating ipilimumab in addition to radiotherapy, grade 3 and 4 adverse events may be significantly higher. In a phase III trial treating 799 patients with metastatic prostate cancer that had progressed after docetaxel treatment, patients were treated via radiotherapy with or without ipilimumab [10]. Three percent of patients treated with radiation therapy alone experienced grade 3 or 4 adverse events, while $26 \%$ treated with radiation plus ipilimumab experienced grade 3 or 4 adverse events. Only 4 deaths occurred (1\%), all in the radiation plus ipilimumab group. Our results are consistent with current data. Grade 3 and 4 adverse events escalate rather rapidly when ipilimumab is combined with additional treatment. This escalation is more pronounced with three or more combination agents.

Our data also showed that grade 3 and 4 toxicity predicted response to treatment and was associated with higher complete and partial responses. This would be theoretically expected, as increased immune-related adverse effects should be elevated when the immune system is stimulated. However, conflicting evidence in past studies both support and refute this finding. In a retrospective analysis of 86 patients treated with ipilimumab, $42 \%$ of patients suffered from autoimmune toxicity and occurrence of toxicity correlated with clinical benefit [11]. Likewise, in a retrospective study consisting of 129 metastatic melanoma patients treated with ipilimumab, the development of any toxicity during treatment was associated with higher progression-free survival [12]. Multivariate analysis showed that absence of toxicity was a prognostic factor for poor overall survival (OS) and progression-free survival. In an analysis of 198 patients in Germany treated with ipilimumab, the occurrence of immune-related adverse events correlated significantly with response to treatment and prolonged OS [13].

On the other hand, a retrospective analysis of 298 patients with melanoma treated with ipilimumab at Memorial Sloan Kettering Cancer Center between 2011 and 2013 demonstrated that OS and time to treatment failure were not affected by the occurrence of immunerelated adverse events or treatment with corticosteroids [14]. No differences in OS or time to treatment failure were found when patients were stratified by immune related adverse events of any grade. These findings were consistent with another retrospective analysis of 855 patients in Italy treated with ipilimumab, in which the investigators concluded that the frequency of immune related adverse events was not associated with response to ipilimumab [15]. Similarly, in an analysis of 209 patients with advanced melanoma treated with ipilimumab, the occurrence of adverse events did not correlate with clinical benefit to ipilimumab [16]. Thus, there is conflicting evidence in the literature regarding a correlation between severe toxicity and response to treatment.

Our data also suggested that grade 3 and 4 adverse events correlated with a better radiological response, a widely accepted marker for treatment efficacy. While data correlating toxicity with radiological response is scarce, in one retrospective analysis of 119 patients with metastatic melanoma treated with anti-CTLA-4 therapy including ipilimumab and tremelimumab, radiological manifestations of immune related adverse events were significantly associated with disease control and improved clinical response [17]. The disease control rate was 55\% for the group with radiological manifestations of immunerelated adverse events. In contrast, only $10 \%$ of patients without radiological manifestations experienced disease control.

The NCI phase I data also showed that low grade toxicity (grade 1 and 2) was moderately associated with the length of ipilimumab therapy, while high grade toxicity (grade 3 and 4) was not associated with duration of treatment. These findings are consistent with the findings of a retrospective analysis of 40 patients in a phase III ipilimumab plus glycoprotein 100 peptide vaccine (gp100) study who later underwent prolonged therapy via re-exposure to ipilimumab [3, 18]. Not only did some patients experience a longer and more effective response after retreatment, side effects were similar to what was observed during initial therapy with no new toxicities. Most of the immune related adverse events were mild (grade 1 and 2), affected the skin and gastrointestinal tract, and were managed with established treatment algorithms. No grade 4 or 5 toxicities occurred in the patients retreated. Furthermore, the study concluded that toxicities seen initially did not predispose to future toxicity during re-exposure treatment, and toxicity was not cumulative between treatments. In another study consisting of 855 patients treated with ipilimumab, 51 patients were retreated upon disease progression [19]. Of these patients, $22 \%$ experienced an adverse event of any grade during the retreatment period, and these were generally mild-tomoderate and similar in frequency compared to induction. Six percent of the patients experienced a grade 3 or 4 adverse event related to ipilimumab upon retreatment. No new types of toxicity occurred.

Our data also suggests that pretreatment lymphocyte count, LDH and albumin levels do not correlate with 
Table 2: Tumor types and study agents across 11 phase 1 clinical trials

\begin{tabular}{ll}
\hline Disease Type & Study Agents \\
\hline Lung Adenocarcinoma & Ipilimumab \\
Ovarian Neoplasms & Gp-100 (Anti-Tumor Vaccine) \\
Chronic Lymphocytic Leukemia & GM-CSF \\
Mantle Cell Lymphoma & Dasatanib \\
Hodgkin's Lymphoma & Cetuximab \\
Myeloma & Rituximab \\
Follicular Lymphoma & Prostvac (Oncolytic Virus) \\
Adenocarcinoma NOS & External Beam Radiation \\
Prostate Cancer & \\
Sarcoma & \\
Renal Cell Carcinoma & \\
Malignant Melanoma & \\
Neuroblastoma & \\
Osteosarcoma & \\
Soft Tissue Neoplasm & \\
Gastrointestinal Stromal Tumor & \\
Head and Neck Carcinoma & \\
Non-Hodgkin's Lymphoma & \\
Myelodysplastic Syndrome & \\
Acute Myeloid Leukemia & \\
\hline
\end{tabular}

toxicity. While phase III data that evaluates these parameters are not available, smaller retrospective analyses evaluate these variables as potential biomarkers to predict ipilimumab efficacy. One study assessed 209 advanced melanoma patients treated with ipilimumab and concluded that the occurrence of adverse events did not correlate with baseline LDH nor relative lymphocyte count [16]. In a retrospective analysis of 183 patients treated with ipilimumab, LDH and neutrophil-to-lymphocyte ratio could differentiate patients into high, moderate and low risk of death, but was not associated with toxicity [12]. Another analysis conducted on 47 advanced melanoma patients also demonstrated a significant association between higher LDH and poor prognosis, but no association between LDH and toxicity [20].

Finally, no associations were found between adverse events and ECOG performance status in our phase I database analysis. Our data did not exhibit trends that would suggest a worse side effect profile in patients with poor performance status, as there was not a significant difference between toxicity in ECOG PS 0 versus $1 / 2$ patients (patients with ECOG PS $>2$ were not included in NCI Phase I clinical trials). Unlike chemotherapy, where tolerability depends on baseline performance status of the patient as therapy is taxing on healthy tissue, immunotherapy side effects are often auto-immune driven which are unpredictable and theoretically unrelated to baseline performance status. Although minimal prospective data exist on the effect of
ECOG performance status in response to ipilimumab, retrospective analyses have suggested associations between ECOG performance status and survival but not with toxicity. For example, a study of 58 metastatic melanoma patients treated with ipilimumab demonstrated that performance status $>1$ was associated with worse survival, as shown by univariate analysis. However, no conclusions were made on an association between toxicity and performance status. In a study consisting of 129 metastatic melanoma patients treated with ipilimumab, univariate analysis showed that a better baseline ECOG performance status (0 versus 1) was associated with improved response to treatment [12]. Again, no conclusions could be made to correlate ECOG performance status with toxicities observed during treatment.

\section{MATERIALS AND METHODS}

For this retrospective study, two authors queried the NCI's Integrated Platform for Agents and Diseases (IPAD) database (version 6.1.0, Rockville, MD) in May 2018 to determine the number of ipilimumab immunotherapy trials alone or in combination in the NCI portfolio at the time. The clinical trials were sorted using search terms of "ipilimumab," "Phase 1 or $1 / 2$," and "complete, closed to accrual," or "closed to accrual and treatment." Once trials were identified, a single reviewer abstracted data from anonymized patient experience adverse reports in 
tabular format. Only ipilimumab-attributed adverse events like symptoms or signs of toxicity, as well as decreased hematological cell counts, blood electrolytes, or proteins were analyzed in this study; therefore, the prevalence of toxicities reported here may differ from primary research publications [21-31]. Events repeating across consecutive cycles were considered a single event for this analysis. Patient demographic, Eastern Cooperative Oncology Group (ECOG) performance status scale score, tumor characteristics and treatment regimens were also abstracted (Table 2). When available, duration of ipilimumab exposure, radiographic responses, disease relapse events and mortality status were recorded. A second reviewer independently provided a data quality-check. The research presented in this article involved the collection and analysis of existing data, documents and records that were publicly available. This research is regarded as exempt from Institutional Review Board oversight.

Categorical data were analyzed using Fisher exact test or Pearson chi-square test and continuous data were analyzed using Wilcoxon rank-sum test $(\alpha=$ 0.05). Correlation between covariates and outcome were presented using Pearson correlation coefficient.

\section{CONCLUSIONS}

Ipilimumab-attributed grade 3 or 4 toxicity is associated with radiological response in this limited series of 11 early phase I clinical trials. Pre-trial performance status, pre-treatment lymphocyte count, lactate dehydrogenase and albumin levels were not associated with ipilimumab-attributed toxicity. The number of added antitumor agents to ipilimumab treatment raised the likelihood of treatment-related adverse events, and duration of treatment was associated with low grade rather than higher grade toxicity.

\section{Author contributions}

AC: conceptualization, investigation, supervision, writing original; TK: investigation, validation, editing; JW: formal analysis, methodology; JWei: formal analysis, methodology; MC: investigation, validation, editing; $\mathrm{CK}$ : conceptualization, investigation, validation, editing.

\section{ACKNOWLEDGMENTS}

Donna Gilbreath at the University of Kentucky Markey Cancer Center's Research Communications Office assisted with preparation of this manuscript.

\section{CONFLICTS OF INTEREST}

The authors have no relationships that they believe could be construed as resulting in an actual, potential, or perceived conflict of interest with regard to this manuscript submitted for review.

\section{FUNDING}

This work was supported by National Institutes of Health [grant number P30 CA177558].

\section{REFERENCES}

1. U.S. National Library of Medicine (Bethesda, MD). 2019. Available from: https://clinicaltrials.gov.

2. Hodi FS, Butler M, Oble DA, Seiden MV, Haluska FG, Kruse A, Macrae S, Nelson M, Canning C, Lowy I, Korman A, Lautz D, Russell S, et al. Immunologic and clinical effects of antibody blockade of cytotoxic T lymphocyteassociated antigen 4 in previously vaccinated cancer patients. Proc Natl Acad Sci U S A. 2008; 105:3005-3010. https://doi.org/10.1073/pnas.0712237105. [PubMed]

3. Hodi FS, O'Day SJ, McDermott DF, Weber RW, Sosman JA, Haanen JB, Gonzalez R, Robert C, Schadendorf D, Hassel JC, Akerley W, van den Eertwegh AJ, Lutzky J, et al. Improved survival with ipilimumab in patients with metastatic melanoma. N Engl J Med. 2010; 363:711-723. https://doi.org/10.1056/NEJMoa1003466. [PubMed]

4. Soldatos TG, Dimitrakopoulou-Strauss A, Larribere L, Hassel JC, Sachpekidis C. Retrospective Side Effect Profiling of the Metastatic Melanoma Combination Therapy Ipilimumab-Nivolumab Using Adverse Event Data. Diagnostics (Basel). 2018; 8. https://doi.org/10.3390/ diagnostics8040076. [PubMed]

5. Sznol M, Ferrucci PF, Hogg D, Atkins MB, Wolter P, Guidoboni M, Lebbe C, Kirkwood JM, Schachter J, Daniels GA, Hassel J, Cebon J, Gerritsen W, et al. Pooled Analysis Safety Profile of Nivolumab and Ipilimumab Combination Therapy in Patients With Advanced Melanoma. J Clin Oncol. 2017; 35:3815-3822. https://doi.org/10.1200/ JCO.2016.72.1167. [PubMed]

6. Bowyer S, Prithviraj P, Lorigan P, Larkin J, McArthur G, Atkinson V, Millward M, Khou M, Diem S, Ramanujam S, Kong B, Liniker E, Guminski A, et al. Efficacy and toxicity of treatment with the anti-CTLA-4 antibody ipilimumab in patients with metastatic melanoma after prior anti-PD-1 therapy. Br J Cancer. 2016; 114:1084-1089. https://doi. org/10.1038/bjc.2016.107. [PubMed]

7. Weber JS, Gibney G, Sullivan RJ, Sosman JA, Slingluff CL Jr, Lawrence DP, Logan TF, Schuchter LM, Nair S, Fecher L, Buchbinder EI, Berghorn E, Ruisi M, et al. Sequential administration of nivolumab and ipilimumab with a planned switch in patients with advanced melanoma (CheckMate 064): an open-label, randomised, phase 2 trial. Lancet Oncol. 2016; 17:943-955. https://doi.org/10.1016/S14702045(16)30126-7. [PubMed]

8. Robert C, Thomas L, Bondarenko I, O'Day S, Weber J, Garbe C, Lebbe C, Baurain JF, Testori A, Grob JJ, Davidson 
N, Richards J, Maio M, et al. Ipilimumab plus dacarbazine for previously untreated metastatic melanoma. N Engl J Med. 2011; 364:2517-2526. https://doi.org/10.1056/ NEJMoa1104621. [PubMed]

9. Reck M, Luft A, Szczesna A, Havel L, Kim SW, Akerley W, Pietanza MC, Wu YL, Zielinski C, Thomas M, Felip E, Gold K, Horn L, et al. Phase III Randomized Trial of Ipilimumab Plus Etoposide and Platinum Versus Placebo Plus Etoposide and Platinum in Extensive-Stage Small-Cell Lung Cancer. J Clin Oncol. 2016; 34:3740-3748. https:// doi.org/10.1200/JCO.2016.67.6601. [PubMed]

10. Kwon ED, Drake CG, Scher HI, Fizazi K, Bossi A, van den Eertwegh AJ, Krainer M, Houede N, Santos R, Mahammedi H, Ng S, Maio M, Franke FA, et al, and CA184-043 Investigators. Ipilimumab versus placebo after radiotherapy in patients with metastatic castration-resistant prostate cancer that had progressed after docetaxel chemotherapy (CA184-043): a multicentre, randomised, double-blind, phase 3 trial. Lancet Oncol. 2014; 15:700-712. https://doi. org/10.1016/S1470-2045(14)70189-5. [PubMed]

11. Dick J, Lang N, Slynko A, Kopp-Schneider A, Schulz C, Dimitrakopoulou-Strauss A, Enk AH, Hassel JC. Use of LDH and autoimmune side effects to predict response to ipilimumab treatment. Immunotherapy. 2016; 8:1033-1044. https://doi.org/10.2217/imt-2016-0083. [PubMed]

12. Khoja L, Atenafu EG, Ye Q, Gedye C, Chappell M, Hogg D, Butler MO, Joshua AM. Real-world efficacy, toxicity and clinical management of ipilimumab treatment in metastatic melanoma. Oncol Lett. 2016; 11:1581-1585. https://doi. org/10.3892/ol.2015.4069. [ubMed]

13. Eigentler TK, Schlaak M, Hassel JC, Loquai C, Stoffels I, Gutzmer R, Patzold S, Mohr P, Keller U, Starz H, Ulrich J, Tsianakas A, Kahler K, et al. Effectiveness and tolerability of ipilimumab: experiences from 198 patients included in a named-patient program in various daily-practice settings and multiple institutions. J Immunother. 2014; 37:374-381. https://doi.org/10.1097/CJ.0000000000000046. [PubMed]

14. Horvat TZ, Adel NG, Dang TO, Momtaz P, Postow MA, Callahan MK, Carvajal RD, Dickson MA, D'Angelo SP, Woo KM, Panageas KS, Wolchok JD, Chapman PB. Immune-Related Adverse Events, Need for Systemic Immunosuppression, and Effects on Survival and Time to Treatment Failure in Patients With Melanoma Treated With Ipilimumab at Memorial Sloan Kettering Cancer Center. J Clin Oncol. 2015; 33:3193-3198. https://doi.org/10.1200/ JCO.2015.60.8448. [PubMed]

15. Ascierto PA, Simeone E, Sileni VC, Pigozzo J, Maio M, Altomonte M, Del Vecchio M, Di Guardo L, Marchetti P, Ridolfi R, Cognetti F, Testori A, Bernengo MG, et al. Clinical experience with ipilimumab $3 \mathrm{mg} / \mathrm{kg}$ : realworld efficacy and safety data from an expanded access programme cohort. J Transl Med. 2014; 12:116. https://doi. org/10.1186/1479-5876-12-116. [PubMed]

16. Martens A, Wistuba-Hamprecht K, Geukes Foppen M, Yuan J, Postow MA, Wong P, Romano E, Khammari A,
Dreno B, Capone M, Ascierto PA, Di Giacomo AM, Maio $\mathrm{M}$, et al. Baseline Peripheral Blood Biomarkers Associated with Clinical Outcome of Advanced Melanoma Patients Treated with Ipilimumab. Clin Cancer Res. 2016; 22:29082918. https://doi.org/10.1158/1078-0432.CCR-15-2412. [PubMed]

17. Bronstein $\mathrm{Y}, \mathrm{Ng} \mathrm{CS}$, Hwu P, Hwu WJ. Radiologic manifestations of immune-related adverse events in patients with metastatic melanoma undergoing anti-CTLA-4 antibody therapy. AJR Am J Roentgenol. 2011; 197:W992W1000. https://doi.org/10.2214/AJR.10.6198. [PubMed]

18. Robert C, Schadendorf D, Messina M, Hodi FS, O'Day $\mathrm{S}$, and MDX010-20 Investigators. Efficacy and safety of retreatment with ipilimumab in patients with pretreated advanced melanoma who progressed after initially achieving disease control. Clin Cancer Res. 2013; 19:22322239. https://doi.org/10.1158/1078-0432.CCR-12-3080. [PubMed]

19. Chiarion-Sileni V, Pigozzo J, Ascierto PA, Simeone E, Maio M, Calabro L, Marchetti P, De Galitiis F, Testori A, Ferrucci PF, Queirolo P, Spagnolo F, Quaglino P, et al. Ipilimumab retreatment in patients with pretreated advanced melanoma: the expanded access programme in Italy. Br J Cancer. 2014; 110:1721-1726. https://doi.org/10.1038/bjc.2014.126. [PubMed]

20. Balatoni T, Ladanyi A, Frohlich G, Czirbesz K, Kovacs P, Panczel G, Bence E, Plotar V, Liszkay G. Biomarkers Associated with Clinical Outcome of Advanced Melanoma Patients Treated with Ipilimumab. Pathol Oncol Res. 2018; 8:pii:E76. https://doi.org/10.1007/s12253-018-0466-9. [PubMed]

21. Schadendorf D, Hodi FS, Robert C, Weber JS, Margolin K, Hamid O, Patt D, Chen TT, Berman DM, Wolchok JD. Pooled Analysis of Long-Term Survival Data From Phase II and Phase III Trials of Ipilimumab in Unresectable or Metastatic Melanoma. J Clin Oncol. 2015; 33:1889-1894. https://doi.org/10.1200/JCO.2014.56.2736. [PubMed]

22. Theoret MR, Arlen PM, Pazdur M, Dahut WL, Schlom J, Gulley JL. Phase I trial of an enhanced prostate-specific antigen-based vaccine and anti-CTLA-4 antibody in patients with metastatic androgen-independent prostate cancer. Clin Genitourin Cancer. 2007; 5:347-350. https:// doi.org/10.3816/CGC.2007.n.017. [PubMed]

23. Bashey A, Medina B, Corringham S, Pasek M, Carrier E, Vrooman L, Lowy I, Solomon SR, Morris LE, Holland HK, Mason JR, Alyea EP, Soiffer RJ, Ball ED. CTLA4 blockade with ipilimumab to treat relapse of malignancy after allogeneic hematopoietic cell transplantation. Blood. 2009; 113:1581-1588. https://doi.org/10.1182/ blood-2008-07-168468. [PubMed]

24. Phase I Study of Ipilimumab (Anti-CTLA-4) in Children and Adolescents With Treatment-Resistant Cancer (NCT01445379). Bethesda (MD): National Library of Medicine; 2011. Available from: https://clinicaltrials.gov.

25. Phase I Study of Dasatinib in Combination With Ipilimumab for Patients With Advanced Gastrointestinal Stromal Tumor 
and Other Sarcomas (NCT01643278). Bethesda (MD): National Library of Medicine; 2012. Available from: https:// clinicaltrials.gov.

26. A Phase Ib Trial of Concurrent Cetuximab (ERBITUX $\left.{ }^{\circledR}\right)$ and Intensity Modulated Radiotherapy (IMRT) With Ipilimumab (YERVOY ${ }^{\circledR}$ ) in Locally Advanced Head and Neck Cancer (NCT01935921). Bethesda (MD): National Library of Medicine; 2013. Available from: https:// clinicaltrials.gov.

27. Ipilimumab and Rituximab in Treating Patients With Relapsed or Refractory B-cell Lymphoma (NCT01729806). Bethesda (MD): National Library of Medicine; 2012. Available from: https://clinicaltrials.gov.

28. A Phase 1 Study of Ipilimumab in Relapsed and Refractory High Risk Myelodysplastic Syndrome and Acute Myeloid Leukemia With Minimal Residual Disease (NCT01757639). Bethesda (MD): National Library of Medicine; 2012. Available from: https://clinicaltrials.gov.
29. Phase I/II Study of Anti-CTLA-4 Monoclonal Antibody (MDX-010) in B-cell Non-Hodgkin's Lymphoma (NCT00287391). US National Library of Medicine. Bethesda (MD): National Library of Medicine; 2012. Available from: https://clinicaltrials.gov.

30. A Pilot Study of Ipilimumab (MDX-CTLA4, MDX-010) in Lymphoma (NCT00047164). Bethesda (MD): National Library of Medicine; 2003. Available from: https:// clinicaltrials.gov.

31. Monoclonal Antibody Therapy in Treating Patients With Ovarian Epithelial Cancer, Melanoma, Acute Myeloid Leukemia, Myelodysplastic Syndrome, or Non-Small Cell Lung Cancer (NCT00039091). Bethesda (MD): National Library of Medicine; 2003. Available from: https:// clinicaltrials.gov. 